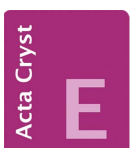

CRYSTALLOGRAPHIC COMMUNICATIONS

ISSN 2056-9890

Received 29 April 2019

Accepted 14 May 2019

Edited by A. V. Yatsenko, Moscow State University, Russia

Keywords: crystal structure; racemate; 2 phenylbutyramide; hydrogen bonds; Hirshfeld surface analysis.

CCDC reference: 1916098

Supporting information: this article has supporting information at journals.iucr.org/e
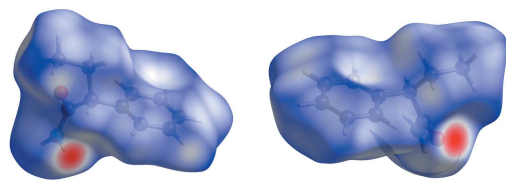

OPEN $\odot$ ACCESS

\section{Crystal structure and Hirshfeld surface analysis of new polymorph of racemic 2-phenylbutyramide}

\author{
Sergei Rigin, ${ }^{\mathrm{a} *}$ Beatrice Armijo, ${ }^{\mathrm{a}}$ Arcadius V. Krivoshein, ${ }^{\mathrm{b}}$ Marina Fonari ${ }^{\mathrm{c}}$ and \\ Tatiana Timofeeva ${ }^{a}$ \\ ${ }^{\mathbf{a}}$ Department of Chemistry, New Mexico Highlands University, Las Vegas, New Mexico, 87701, USA, ${ }^{\mathbf{b}}$ Department of \\ Physical \& Applied Sciences, University of Houston - Clear Lake, 2700 Bay Area Boulevard, Houston, TX 77058, USA, \\ and 'Institute of Applied Physics, Academy str., 5 MD2028, Chisinau, Moldova. ${ }^{*}$ Correspondence e-mail: \\ rigindale@gmail.com
}

A new polymorph of the title compound, $\mathrm{C}_{10} \mathrm{H}_{13} \mathrm{NO}$, was obtained by recrystallization of the commercial product from a water/ethanol mixture (1:1 $v / v)$. Crystals of the previously reported racemic and homochiral forms of 2-phenylbutyramide were grown from water-acetonitrile solution in 1:1 volume ratio [Khrustalev et al. (2014). Cryst. Growth Des. 14, 3360-3369]. While the previously reported racemic and enantiopure forms of the title compound adopt very similar supramolecular structures (hydrogen-bonded ribbons), the new racemic polymorph is stabilized by a single $\mathrm{N}-\mathrm{H} \cdots \mathrm{O}$ hydrogen bond that links molecules into chains along the $c$-axis direction with an antiparallel (centrosymmetric) packing in the crystal. Hirshfeld molecular surface analysis was employed to compare the intermolecular interactions in the polymorphs of the title compound.

\section{Chemical context}

Many drugs exist in several polymorphic modifications (Bernstein, 2011; Brittain, 2009). For example, a second polymorph, II, was reported (Vishweshwar et al., 2005) in 2005 for aspirin, one of the most widely consumed medications; this was similar in structure to the original form I (Wheatley, 1964) and was widely discussed (Bond et al., 2007, 2011). The third ambient polymorph of aspirin, crystallized from the melt, was described recently using a combination of X-ray powder diffraction analysis and crystal structure prediction algorithms (Shtukenberg et al., 2017). Paracetamol, one of the most frequently used antipyretic and analgesic drugs, is known in three crystal modifications: a monoclinic (form I) (Haisa et al., 1976), an orthorhombic (form II) (Haisa et al., 1974), and an unstable phase (form III), which can only be stabilized under certain conditions (Burger \& Ramberger, 1979). The nonsteroidal anti-inflammatory drug mefenamic acid is known to exist as dimorphs (I and II) and a metastable polymorph obtained during co-crystallization experiments (SeethaLekshmi \& Guru Row, 2012). The existence of three different polymorphic forms has been reported for anhydrous carbamazepine, an anticonvulsant drug (Rustichelli et al., 2000). We previously reported (Khrustalev et al., 2014) two polymorphs of $\alpha$-methyl- $\alpha$-phenylsuccinimide (3-methyl-3-phenylpyrrolidine-2,5-dione), the $N$-demethylated metabolite of the anticonvulsant methsuximide. Herein, we report on the crystal structure and the Hirshfeld surface analysis of a new polymorph of the title compound, obtained by recrystallization of 
the commercial product (Alfa Aesar, stock No. A18501) from a water-ethanol (1:1) solution. Crystals of the previously reported racemic and homochiral forms of 2-phenylbutyramide were grown from water-acetonitrile solution in a 1:1 volume ratio (Khrustalev et al., 2014).<smiles>CCC(C(N)=O)c1ccccc1</smiles>

\section{Structural commentary}

A view of the molecule of the new polymorph (henceforth referred to as $r a c-2$ ) is illustrated in Fig. 1a. In the molecule, the rotation of the amide group around the $\mathrm{C} 7-\mathrm{C} 10$ bond is characterized by an $\mathrm{N} 1-\mathrm{C} 10-\mathrm{C} 7-\mathrm{C} 8$ torsion angle of $-141.14(9)^{\circ}$, thus corresponding in conformation to the previously reported polymorph (further notated as rac-1, space group $C 2 / c$ ), where the torsion angle is $-130.06(9)^{\circ}$, and one of two conformers in the $R$-enantiomer [space group $P 1$, torsion angle $=-144.08(13)^{\circ} ;$ Khrustalev et al., 2014). The overlay diagram for the two racemic forms shown in Fig. $1 b$ shows the almost perfect fit (r.m.s. deviation $=0.263 \AA$ ). The bond lengths in the molecule are in line with those of reported analogues (CSD version 5.40, last update November 2018; Groom et al., 2016).

\section{Supramolecular features}

Molecule of the title compound contain one amino group as a potential double hydrogen-bond donor and one carbonyl

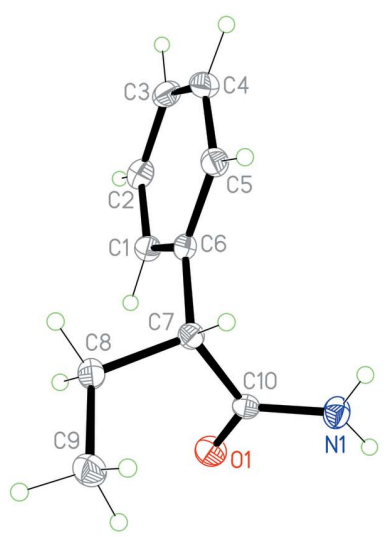

(a)

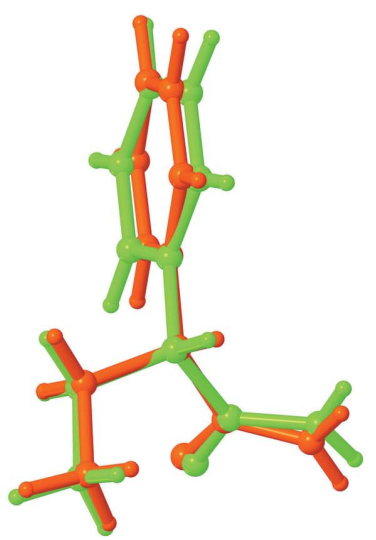

(b)
Figure 1

(a) View of the molecular structure of the title rac-2 polymorph with the atom labelling. Displacement ellipsoids are drawn at the $50 \%$ probability level. (b) Superposition of the rac-1 (red) and rac-2 (green) polymorphs.
Table 1

Hydrogen-bond geometry $\left(\AA,^{\circ}\right)$.

\begin{tabular}{lllll}
\hline$D-\mathrm{H} \cdots A$ & $D-\mathrm{H}$ & $\mathrm{H} \cdots A$ & $D \cdots A$ & $D-\mathrm{H} \cdots A$ \\
\hline $\mathrm{N} 1-\mathrm{H} 1 B \cdots \mathrm{O} 1^{\mathrm{i}}$ & $0.895(15)$ & $2.071(15)$ & $2.9533(13)$ & $168.4(13)$ \\
\hline
\end{tabular}

Symmetry code: (i) $x,-y+\frac{1}{2}, z+\frac{1}{2}$.

group capable of acting as a multiple hydrogen-bond acceptor. Contrary to the previously reported $r a c-1$ and two enantiomeric forms (Khrustalev et al., 2014), where the amino group acted as double hydrogen-bond donor while the carbonyl oxygen atom acted as a double hydrogen-bond acceptor being involved in two $\mathrm{N}-\mathrm{H} \cdots \mathrm{O}$ hydrogen bonds leading to the formation of supramolecular ribbons, in rac-2 only one hydrogen atom of the amino group is involved in a single $\mathrm{N}-$ $\mathrm{H} \cdots \mathrm{O}$ hydrogen bond (Table 1). This hydrogen bond links molecules related by the glide plane into chains along the $c$ axis direction (Fig. 2). The packing of the chains obeys inversion symmetry with only van der Waals contacts between the chains (Fig. 3). In spite of the fewer number of strong directed intermolecular interactions in the crystal, the structure of rac-2 is characterized by a more effective crystal packing of the single chains, compared to the packing of ribbons in rac-1 and in the enantiomers, which follows from the higher value of the crystal density (calculated as $1.227 \mathrm{~g} \mathrm{~cm}^{-3}$; Table 2) compared with values of $1.160 \mathrm{~g} \mathrm{~cm}^{-3}$

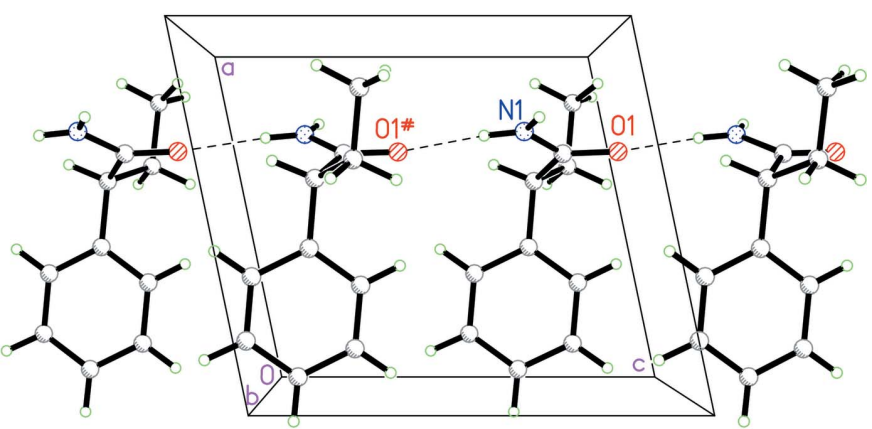

Figure 2

View of a hydrogen-bonded chain.

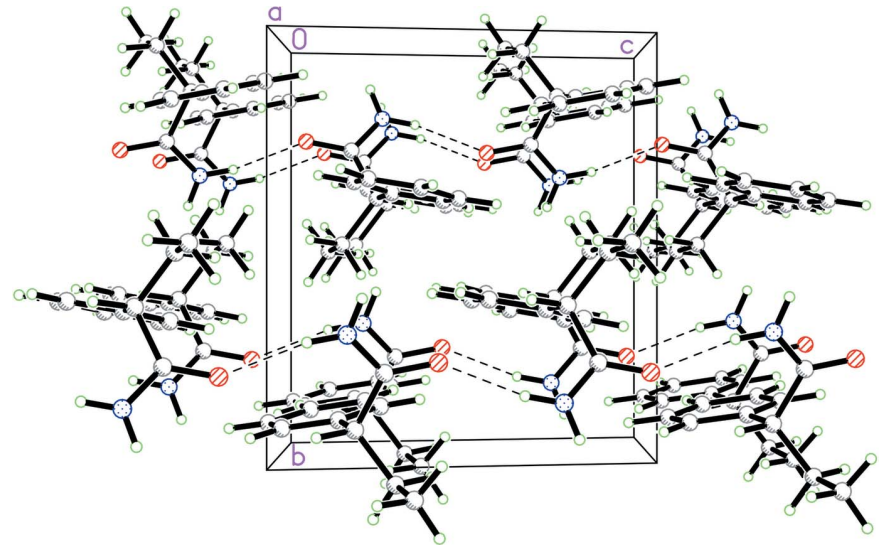

Figure 3

The crystal packing of the rac-2 polymorph. 
Table 2

Crystal lattice energies $\left(\mathrm{kJ} \mathrm{mol}^{-1}\right)$ for racemic 2-phenylbutyramide polymorphs computed using $A A$-CLP software.

\begin{tabular}{lllllll}
\hline Polymorph & $E_{\text {electrostatic }}$ & $E_{\text {polarization }}$ & $E_{\text {dispersion }}$ & $E_{\text {exchange-repulsion }}$ & $E_{\text {total }}$ & ${\text { Crystal density }\left(\mathrm{g} \mathrm{cm}^{-3}\right)}$ \\
rac-1 & $-35.6^{a}$ & $-27.6^{a}$ & $-100.4^{a}$ & $55.0^{a}$ & $-108.6^{a}$ & $1.160^{b}$ \\
rac-2 & -33.9 & -28.3 & -107.9 & 53.8 & -116.3 & 1.227 \\
\hline
\end{tabular}

Notes: ${ }^{a}$ from Krivoshein et al. (2018); ${ }^{b}$ from Khrustalev et al. (2014).

for $r a c-1$ and $1.188 \mathrm{~g} \mathrm{~cm}^{-3}$ and $1.189 \mathrm{~g} \mathrm{~cm}^{-3}$ for the $R$ - and $S$ enantiomers (Khrustalev et al., 2014).

\section{Hirshfeld surface analysis and calculation of crystal lattice energies}

Crystal Explorer (Wolff et al., 2012) was used to generate the Hirshfeld surfaces (Hirshfeld, 1977). The total $d_{\text {norm }}$ surfaces for polymorphs rac-2 and rac-1 are shown in Figs. 4 and 5, respectively, in which the red spots correspond to the most significant $\mathrm{N}-\mathrm{H} \cdots \mathrm{O}$ interactions in the crystal (Table 1$)$. The surface diagram unambiguously shows that there are fewer active binding sites in $r a c-2$ in comparison to rac- 1 . The twodimensional fingerprint plots from the Hirshfeld surface analysis (Spackman \& Jayatilaka, 2009) allows the intermolecular interactions to be analysed in detail and for even rather subtle differences between polymorphic systems to be quantified (Bernstein, 2011). The two-dimensional fingerprint plots for rac-2 and rac-1 are shown in Figs. 6 and 7, respectively. They clearly indicate the different distribution of interactions for a single molecule in the two structures. Decomposition of the full fingerprint plot for $r a c-2$ shows five principle types of interactions that include $\mathrm{H} \cdots \mathrm{H}, \mathrm{H} \cdots \mathrm{C} /$ $\mathrm{C} \cdots \mathrm{H}, \quad \mathrm{H} \cdots \mathrm{O} / \mathrm{O} \cdots \mathrm{H}, \quad \mathrm{H} \cdots \mathrm{N} / \mathrm{N} \cdots \mathrm{H}, \quad$ and $\mathrm{C} \cdots \mathrm{O} / \mathrm{O} \cdots \mathrm{C}$
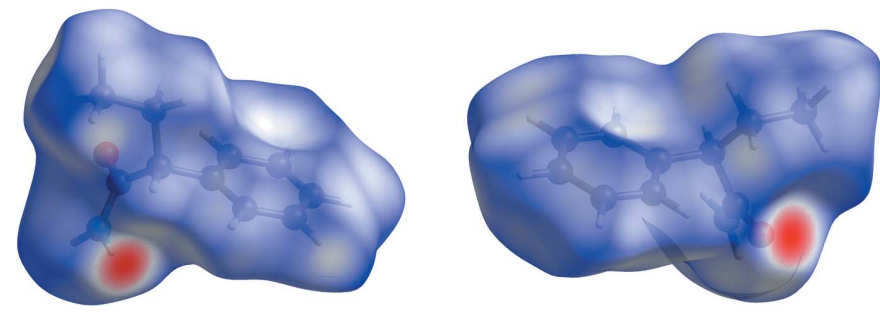

Figure 4

Hirshfeld surface for the $r a c$-2 polymorph plotted over $d_{\text {norm }}$ in the range -0.4994 to 1.0567 a.u.
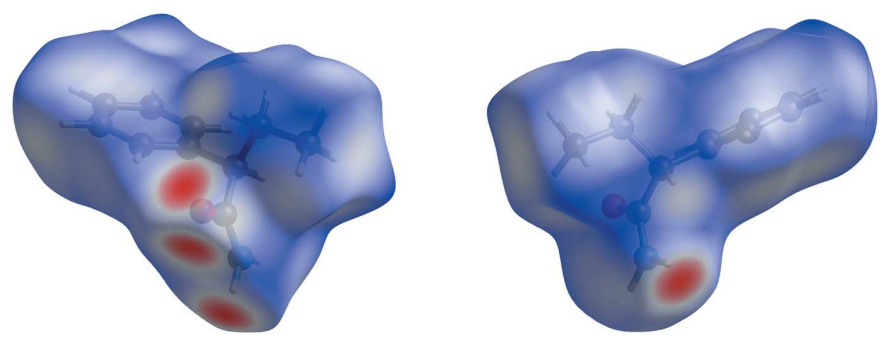

Figure 5

Hirshfeld surface for the rac-1 polymorph plotted over $d_{\text {norm }}$ in the range -0.5239 to 1.3882 a.u. contacts in decreasing order (Fig. 6). For the rac-1 polymorph, the set includes only four types of interactions, viz. $\mathrm{H} \cdots \mathrm{H}$, $\mathrm{H} \cdots \mathrm{C} / \mathrm{C} \cdots \mathrm{H}, \quad \mathrm{H} \cdots \mathrm{O} / \mathrm{O} \cdots \mathrm{H}$ and $\mathrm{H} \cdots \mathrm{N} / \mathrm{N} \cdots \mathrm{H}$ contacts (Fig. 7). The predominant interactions in both cases are $\mathrm{H} \cdot \mathrm{H}$, constituting $65.7 \%$ in $\mathrm{rac}-2$ and $67.3 \%$ in $\mathrm{rac}-1$. With a significantly less contribution, the next most important interactions are $\mathrm{H} \cdots \mathrm{C} / \mathrm{C} \cdots \mathrm{H}$, contributing $19.6 \%$ in both cases, and being slightly asymmetric in shape in favour of (internal)C..H(external) contacts for both polymorphs. The
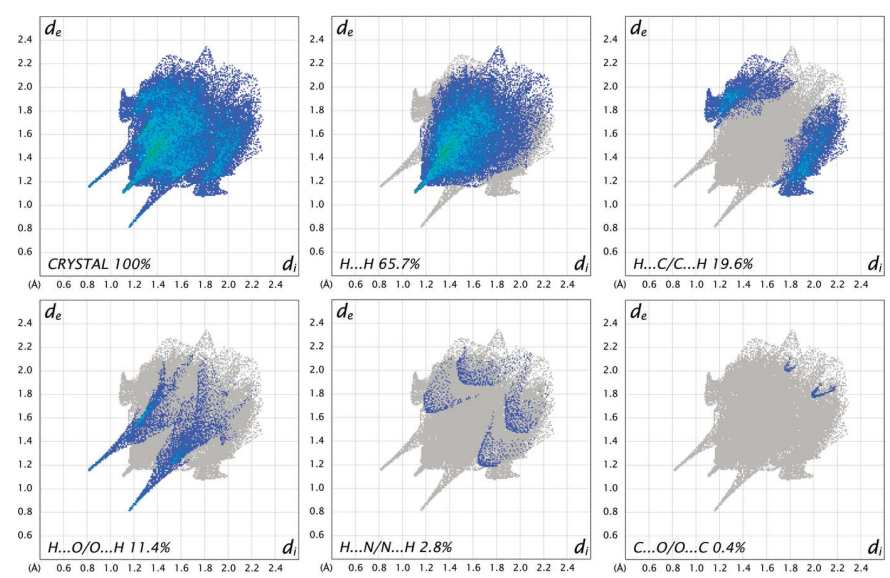

Figure 6

The full two-dimensional fingerprint plots for the rac-2 polymorph, showing all interactions, and delineated into $\mathrm{H} \cdots \mathrm{H}, \mathrm{H} \cdots \mathrm{C} / \mathrm{C} \cdots \mathrm{H}$, $\mathrm{H} \cdots \mathrm{O} / \mathrm{O} \cdots \mathrm{H}, \mathrm{H} \cdots \mathrm{N} / \mathrm{N} \cdots \mathrm{H}, \mathrm{C} \cdots \mathrm{O} / \mathrm{O} \cdots \mathrm{C}$ interactions. The $d_{\mathrm{i}}$ and $d_{\mathrm{e}}$ values are the closest internal and external distances (in $\AA$ ) from given points on the Hirshfeld surface.
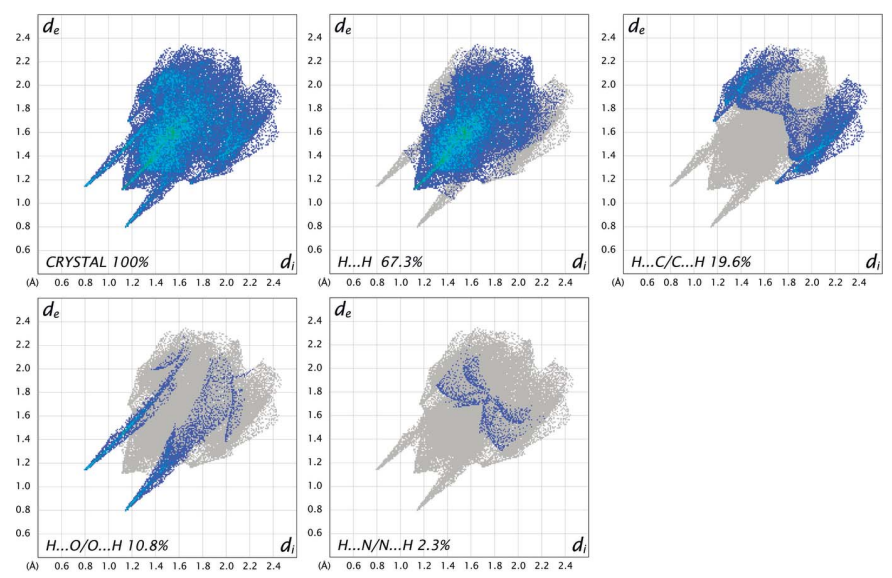

Figure 7

The full two-dimensional fingerprint plots for the rac-1 polymorph, showing all interactions, and delineated into $\mathrm{H} \cdots \mathrm{H}, \mathrm{H} \cdots \mathrm{C} / \mathrm{C} \cdots \mathrm{H}$, $\mathrm{H} \cdots \mathrm{O} / \mathrm{O} \cdots \mathrm{H}$ and $\mathrm{H} \cdots \mathrm{N} / \mathrm{N} \cdots \mathrm{H}$ interactions. The $d_{\mathrm{i}}$ and $d_{\mathrm{e}}$ values are the closest internal and external distances (in $\AA$ ) from given points on the Hirshfeld surface. 
Table 3

Experimental details.

\begin{tabular}{|c|c|}
\hline Crystal data & \\
\hline Chemical formula & $\mathrm{C}_{10} \mathrm{H}_{13} \mathrm{NO}$ \\
\hline$M_{\mathrm{r}}$ & 163.21 \\
\hline Crystal system, space group & Monoclinic, $P 2_{1} / c$ \\
\hline Temperature (K) & 100 \\
\hline$a, b, c(\AA)$ & $8.575(2), 10.746(3), 9.798(3)$ \\
\hline$\beta\left({ }^{\circ}\right)$ & $101.811(3)$ \\
\hline$V\left(\AA^{3}\right)$ & $883.8(4)$ \\
\hline$Z$ & 4 \\
\hline Radiation type & Мo $K \alpha$ \\
\hline$\mu\left(\mathrm{mm}^{-1}\right)$ & 0.08 \\
\hline Crystal size $(\mathrm{mm})$ & $0.15 \times 0.1 \times 0.1$ \\
\hline Data collection & \\
\hline Diffractometer & Bruker APEXII CCD \\
\hline Absorption correction & $\begin{array}{l}\text { Multi-scan (SADABS; Bruker, } \\
\text { 2004) }\end{array}$ \\
\hline$T_{\min }, T_{\max }$ & $0.674,0.746$ \\
\hline $\begin{array}{l}\text { No. of measured, independent and } \\
\text { observed }[I>2 \sigma(I)] \text { reflections }\end{array}$ & $9961,2240,1914$ \\
\hline$R_{\text {int }}$ & 0.038 \\
\hline$(\sin \theta / \lambda)_{\max }\left(\AA^{-1}\right)$ & 0.671 \\
\hline Refinement & \\
\hline$R\left[F^{2}>2 \sigma\left(F^{2}\right)\right], w R\left(F^{2}\right), S$ & $0.040,0.110,1.08$ \\
\hline No. of reflections & 2240 \\
\hline No. of parameters & 161 \\
\hline $\mathrm{H}$-atom treatment & All H-atom parameters refined \\
\hline$\Delta \rho_{\max }, \Delta \rho_{\min }\left(\mathrm{e} \AA^{-3}\right)$ & $0.31,-0.27$ \\
\hline
\end{tabular}

Computer programs: APEX2 and SAINT (Bruker, 2004), SHELXT (Sheldrick, 2015a), SHELXL (Sheldrick, 2015b) and OLEX2 (Dolomanov et al., 2009).

directed $\mathrm{H} \cdots \mathrm{O} / \mathrm{O} \cdots \mathrm{H}$ contacts constitute $11.4 \%$ for $\mathrm{rac}-2$ and $10.8 \%$ for rac-1, with slight a asymmetry in favour of (internal) $\mathrm{O} \cdots \mathrm{H}$ (external) contacts for both polymorphs.

The Hirshfeld surface analysis confirms the decisive role of $\mathrm{H}$-contacts that include hydrogen bonding and van der Waals interactions in the crystal packing. The crystal-lattice energies (Table 2) were calculated from the atomic coordinates obtained in the single-crystal X-ray diffraction experiments using the atom-atom force field with subdivision of the interaction energies into Coulombic, polarization, London dispersion, and Pauli repulsion components ( $A A-C L P$; Gavezzotti, 2011, 2013) implemented in the CLP-PIXEL computer program package (version 3.0, available from www.angelogavezzotti.it). These show that the rac-2 polymorph is more stable in terms of two criteria: total crystal energy and crystal density.

\section{Database survey}

The Cambridge Structural Database (CSD version 5.40, last update November 2018; Groom et al., 2016) includes crystallographic data for the $R$ - and $S$-enantiomers of 2-phenylbutyramide (VOQGUF and VOQHAM, space group $P 1$; Khrustalev et al., 2014) and the racemic form (VOQHEQ, space group $C 2 / c$; Khrustalev et al., 2014). As mentioned above, the conformations of two rac-polymorphs are quite similar, while the crystal packing differs significantly with more efficient crystal packing for the rac-2 polymorph reported here.

\section{Crystallization}

Crystals were obtain by the slow evaporation approach. $0.5 \mathrm{~g}$ of 2-phenylbutyramide (Alfa Aesar, stock No. A18501) were dissolved with extensive vortexing in $3 \mathrm{~mL}$ of a water/ethanol mixture (1:1 v/v) and left at room temperature (293-295 K) for six weeks. Block-shaped crystals formed on the walls of the vessel.

\section{Refinement}

Crystal data, data collection and structure refinement details are summarized in Table 3.

\section{Funding information}

Funding for this research was provided by: NSF DMR 1523611 (PREM) and Welch Foundation (Departmental Grant; award No. BC-0022) .

\section{References}

Bernstein, J. (2011). Cryst. Growth Des. 11, 632-650.

Bond, A. D., Boese, R. \& Desiraju, G. R. (2007). Angew. Chem. Int. Ed. 46, 618-622.

Bond, A. D., Solanko, K. A., Parsons, S., Redder, S. \& Boese, R. (2011). CrystEngComm, 13, 399-401.

Brittain, H. G. (2009). Polymorphism in pharmaceutical solids. Informa, Healthcare. NY.

Bruker (2004). SAINT, APEX2 and SADABS. Bruker AXS Inc., Madison, Wisconsin, USA.

Burger, A. \& Ramberger, R. (1979). Mikrochim. Acta, 72, 273-316.

Dolomanov, O. V., Bourhis, L. J., Gildea, R. J., Howard, J. A. K. \& Puschmann, H. (2009). J. Appl. Cryst. 42, 339-341.

Gavezzotti, A. (2011). New J. Chem. 35, 1360-1368.

Gavezzotti, A. (2013). New J. Chem. 37, 2110-2119.

Groom, C. R., Bruno, I. J., Lightfoot, M. P. \& Ward, S. C. (2016). Acta Cryst. B72, 171-179.

Haisa, M., Kashino, S., Kawai, R. \& Maeda, H. (1976). Acta Cryst. B32, 1283-1285.

Haisa, M., Kashino, S. \& Maeda, H. (1974). Acta Cryst. B30, 25102512.

Hirshfeld, F. L. (1977). Theor. Chim. Acta, 44, 129-138.

Khrustalev, V. N., Sandhu, B., Bentum, S., Fonari, A., Krivoshein, A. V. \& Timofeeva, T. V. (2014). Cryst. Growth Des. 14, 3360-3369.

Krivoshein, A. V., Lindeman, S. V., Bentum, S., Averkiev, B. B., Sena, V. \& Timofeeva, T. V. (2018). Z. Kristallogr. Cryst. Mat. 233, 781793.

Rustichelli, C., Gamberini, G., Ferioli, V., Gamberini, M. C., Ficarra, R. \& Tommasini, S. (2000). J. Pharm. Biomed. Anal. 23, 41-54.

SeethaLekshmi, S. \& Guru Row, T. N. (2012). Cryst. Growth Des. 12 , 4283-4289.

Sheldrick, G. M. (2015a). Acta Cryst. A71, 3-8.

Sheldrick, G. M. (2015b). Acta Cryst. C71, 3-8.

Shtukenberg, A. G., Hu, C. T., Zhu, Q., Schmidt, M. U., Xu, W., Tan, M. \& Kahr, B. (2017). Cryst. Growth Des. 17, 3562-3566.

Spackman, M. A. \& Jayatilaka, D. (2009). CrystEngComm, 11, 19-32.

Vishweshwar, P., McMahon, J. A., Oliveira, M., Peterson, M. L. \& Zaworotko, M. J. (2005). J. Am. Chem. Soc. 127, 16802-16803.

Wheatley, P. J. (1964). J. Chem. Soc. pp. 6036-6048.

Wolff, S. K., Grimwood, D. J., McKinnon, J. J., Turner, M. J., Jayatilaka, D. \& Spackman, M. A. (2012). Crystal Explorer. University of Western Australia, Australia. 


\section{supporting information}

Acta Cryst. (2019). E75, 826-829 [https://doi.org/10.1107/S2056989019007011]

Crystal structure and Hirshfeld surface analysis of new polymorph of racemic 2phenylbutyramide

Sergei Rigin, Beatrice Armijo, Arcadius V. Krivoshein, Marina Fonari and Tatiana Timofeeva

Computing details

Data collection: SAINT (Bruker, 2004); cell refinement: APEX2 (Bruker, 2004); data reduction: SAINT (Bruker, 2004);

program(s) used to solve structure: SHELXT (Sheldrick, 2015a); program(s) used to refine structure: SHELXL

(Sheldrick, 2015b); molecular graphics: OLEX2 (Dolomanov et al., 2009); software used to prepare material for publication: OLEX2 (Dolomanov et al., 2009).

2-Phenylbutyramide

Crystal data

$\mathrm{C}_{10} \mathrm{H}_{13} \mathrm{NO}$

$M_{r}=163.21$

Monoclinic, $P 2{ }_{1} / c$

$a=8.575$ (2) $\AA$

$b=10.746(3) \AA$

$c=9.798(3) \AA$

$\beta=101.811(3)^{\circ}$

$V=883.8(4) \AA^{3}$

$Z=4$

\section{Data collection}

Bruker APEXII CCD diffractometer

$\varphi$ and $\omega$ scans

Absorption correction: multi-scan

(SADABS; Bruker, 2004)

$T_{\min }=0.674, T_{\max }=0.746$

9961 measured reflections

\section{Refinement}

Refinement on $F^{2}$

Least-squares matrix: full

$R\left[F^{2}>2 \sigma\left(F^{2}\right)\right]=0.040$

$w R\left(F^{2}\right)=0.110$

$S=1.08$

2240 reflections

161 parameters

0 restraints
$F(000)=352$

$D_{\mathrm{x}}=1.227 \mathrm{Mg} \mathrm{m}^{-3}$

Mo $K \alpha$ radiation, $\lambda=0.71073 \AA$

Cell parameters from 3598 reflections

$\theta=2.4-30.1^{\circ}$

$\mu=0.08 \mathrm{~mm}^{-1}$

$T=100 \mathrm{~K}$

Block, colourless

$0.15 \times 0.1 \times 0.1 \mathrm{~mm}$

2240 independent reflections

1914 reflections with $I>2 \sigma(I)$

$R_{\text {int }}=0.038$

$\theta_{\max }=28.5^{\circ}, \theta_{\min }=2.4^{\circ}$

$h=-11 \rightarrow 10$

$k=-14 \rightarrow 14$

$l=-13 \rightarrow 13$

Primary atom site location: dual

Hydrogen site location: difference Fourier map

All H-atom parameters refined

$w=1 /\left[\sigma^{2}\left(F_{\mathrm{o}}^{2}\right)+(0.0614 P)^{2}+0.1474 P\right]$

where $P=\left(F_{\mathrm{o}}^{2}+2 F_{\mathrm{c}}^{2}\right) / 3$

$(\Delta / \sigma)_{\max }<0.001$

$\Delta \rho_{\max }=0.31$ e $\AA^{-3}$

$\Delta \rho_{\min }=-0.27$ e $\AA^{-3}$ 


\section{Special details}

Geometry. All esds (except the esd in the dihedral angle between two 1.s. planes) are estimated using the full covariance matrix. The cell esds are taken into account individually in the estimation of esds in distances, angles and torsion angles; correlations between esds in cell parameters are only used when they are defined by crystal symmetry. An approximate (isotropic) treatment of cell esds is used for estimating esds involving l.s. planes.

Fractional atomic coordinates and isotropic or equivalent isotropic displacement parameters $\left(\AA^{2}\right)$

\begin{tabular}{lllll}
\hline & $x$ & $y$ & $z$ & $U_{\text {iso }} / U_{\text {eq }}$ \\
\hline O1 & $0.32919(9)$ & $0.23775(7)$ & $0.06137(7)$ & $0.0216(2)$ \\
N1 & $0.27946(11)$ & $0.17768(8)$ & $0.26886(9)$ & $0.0192(2)$ \\
H1A & $0.2469(17)$ & $0.1033(15)$ & $0.2342(15)$ & $0.031(4)^{*}$ \\
H1B & $0.2895(17)$ & $0.1927(14)$ & $0.3601(15)$ & $0.028(3)^{*}$ \\
C1 & $0.68011(12)$ & $0.34374(9)$ & $0.20614(10)$ & $0.0179(2)$ \\
H1 & $0.6296(16)$ & $0.3276(12)$ & $0.1099(14)$ & $0.021(3)^{*}$ \\
C2 & $0.84492(13)$ & $0.33963(10)$ & $0.24848(11)$ & $0.0206(2)$ \\
H2 & $0.9097(17)$ & $0.3192(13)$ & $0.1809(14)$ & $0.027(3)^{*}$ \\
C3 & $0.91672(13)$ & $0.36033(10)$ & $0.38704(11)$ & $0.0217(2)$ \\
H3 & $1.0322(19)$ & $0.3568(14)$ & $0.4177(15)$ & $0.035(4)^{*}$ \\
C4 & $0.82161(13)$ & $0.38440(10)$ & $0.48320(11)$ & $0.0219(2)$ \\
H4 & $0.8698(17)$ & $0.3979(13)$ & $0.5822(15)$ & $0.027(3)^{*}$ \\
C5 & $0.65680(13)$ & $0.38737(9)$ & $0.44137(10)$ & $0.0184(2)$ \\
H5 & $0.5908(15)$ & $0.4038(12)$ & $0.5099(13)$ & $0.017(3)^{*}$ \\
C6 & $0.58384(12)$ & $0.36770(8)$ & $0.30233(10)$ & $0.0151(2)$ \\
C7 & $0.40388(12)$ & $0.37876(9)$ & $0.25694(10)$ & $0.0150(2)$ \\
H7 & $0.3586(15)$ & $0.3913(12)$ & $0.3424(13)$ & $0.019(3)^{*}$ \\
C8 & $0.35882(12)$ & $0.49138(9)$ & $0.16087(11)$ & $0.0189(2)$ \\
H8A & $0.4084(18)$ & $0.5661(13)$ & $0.2142(15)$ & $0.030(4)^{*}$ \\
H8B & $0.4098(16)$ & $0.4803(12)$ & $0.0771(14)$ & $0.024(3)^{*}$ \\
C9 & $0.18019(13)$ & $0.50974(11)$ & $0.11476(12)$ & $0.0226(2)$ \\
H9A & $0.1318(18)$ & $0.4394(14)$ & $0.0522(15)$ & $0.033(4)^{*}$ \\
H9B & $0.1575(19)$ & $0.5862(15)$ & $0.0573(16)$ & $0.039(4)^{*}$ \\
H9C & $0.1253(18)$ & $0.5153(14)$ & $0.1957(16)$ & $0.034(4)^{*}$ \\
C10 & $0.33405(11)$ & $0.25883(9)$ & $0.18573(10)$ & $0.0148(2)$ \\
& & & & \\
\end{tabular}

Atomic displacement parameters $\left(\AA^{2}\right)$

\begin{tabular}{lllllll}
\hline & $U^{11}$ & $U^{22}$ & $U^{33}$ & $U^{12}$ & $U^{13}$ & $U^{23}$ \\
\hline O1 & $0.0261(4)$ & $0.0250(4)$ & $0.0141(4)$ & $-0.0021(3)$ & $0.0046(3)$ & $-0.0035(3)$ \\
N1 & $0.0241(5)$ & $0.0158(4)$ & $0.0184(4)$ & $-0.0028(3)$ & $0.0060(3)$ & $-0.0006(3)$ \\
C1 & $0.0198(5)$ & $0.0181(5)$ & $0.0155(4)$ & $-0.0020(4)$ & $0.0026(4)$ & $-0.0003(3)$ \\
C2 & $0.0202(5)$ & $0.0185(5)$ & $0.0244(5)$ & $-0.0016(4)$ & $0.0073(4)$ & $-0.0004(4)$ \\
C3 & $0.0168(5)$ & $0.0182(5)$ & $0.0279(5)$ & $-0.0025(4)$ & $-0.0004(4)$ & $0.0011(4)$ \\
C4 & $0.0259(6)$ & $0.0191(5)$ & $0.0177(5)$ & $-0.0025(4)$ & $-0.0025(4)$ & $-0.0003(4)$ \\
C5 & $0.0227(5)$ & $0.0160(5)$ & $0.0162(5)$ & $-0.0006(4)$ & $0.0034(4)$ & $-0.0003(3)$ \\
C6 & $0.0167(5)$ & $0.0118(4)$ & $0.0163(4)$ & $-0.0012(3)$ & $0.0022(3)$ & $0.0010(3)$ \\
C7 & $0.0163(5)$ & $0.0159(5)$ & $0.0129(4)$ & $-0.0007(3)$ & $0.0035(3)$ & $-0.0002(3)$ \\
C8 & $0.0187(5)$ & $0.0176(5)$ & $0.0202(5)$ & $-0.0003(4)$ & $0.0036(4)$ & $0.0036(4)$
\end{tabular}




\begin{tabular}{lllllll}
$\mathrm{C} 9$ & $0.0196(5)$ & $0.0231(5)$ & $0.0244(5)$ & $0.0031(4)$ & $0.0028(4)$ & $0.0036(4)$ \\
$\mathrm{C} 10$ & $0.0131(4)$ & $0.0158(4)$ & $0.0150(4)$ & $0.0012(3)$ & $0.0020(3)$ & $-0.0003(3)$ \\
\hline
\end{tabular}

Geometric parameters $\left(\AA,{ }^{o}\right)$

\begin{tabular}{|c|c|c|c|}
\hline $\mathrm{O} 1-\mathrm{C} 10$ & $1.2316(12)$ & $\mathrm{C} 5-\mathrm{H} 5$ & $0.979(12)$ \\
\hline $\mathrm{N} 1-\mathrm{H} 1 \mathrm{~A}$ & $0.890(16)$ & $\mathrm{C} 5-\mathrm{C} 6$ & $1.3940(14)$ \\
\hline $\mathrm{N} 1-\mathrm{H} 1 \mathrm{~B}$ & $0.895(15)$ & $\mathrm{C} 6-\mathrm{C} 7$ & $1.5206(14)$ \\
\hline $\mathrm{N} 1-\mathrm{C} 10$ & $1.3414(13)$ & $\mathrm{C} 7-\mathrm{H} 7$ & $1.002(12)$ \\
\hline $\mathrm{C} 1-\mathrm{H} 1$ & $0.970(13)$ & $\mathrm{C} 7-\mathrm{C} 8$ & $1.5333(14)$ \\
\hline $\mathrm{C} 1-\mathrm{C} 2$ & $1.3898(15)$ & $\mathrm{C} 7-\mathrm{C} 10$ & $1.5282(13)$ \\
\hline $\mathrm{C} 1-\mathrm{C} 6$ & $1.3982(14)$ & $\mathrm{C} 8-\mathrm{H} 8 \mathrm{~A}$ & $1.004(14)$ \\
\hline $\mathrm{C} 2-\mathrm{H} 2$ & $0.973(14)$ & $\mathrm{C} 8-\mathrm{H} 8 \mathrm{~B}$ & $1.013(13)$ \\
\hline $\mathrm{C} 2-\mathrm{C} 3$ & $1.3891(15)$ & $\mathrm{C} 8-\mathrm{C} 9$ & $1.5187(15)$ \\
\hline $\mathrm{C} 3-\mathrm{H} 3$ & $0.975(16)$ & C9-H9A & $1.007(15)$ \\
\hline $\mathrm{C} 3-\mathrm{C} 4$ & $1.3909(16)$ & C9-H9B & $0.992(16)$ \\
\hline $\mathrm{C} 4-\mathrm{H} 4$ & $0.984(14)$ & $\mathrm{C} 9-\mathrm{H} 9 \mathrm{C}$ & $1.004(15)$ \\
\hline $\mathrm{C} 4-\mathrm{C} 5$ & $1.3890(15)$ & & \\
\hline $\mathrm{H} 1 \mathrm{~A}-\mathrm{N} 1-\mathrm{H} 1 \mathrm{~B}$ & $120.1(13)$ & $\mathrm{C} 6-\mathrm{C} 7-\mathrm{H} 7$ & $108.0(7)$ \\
\hline $\mathrm{C} 10-\mathrm{N} 1-\mathrm{H} 1 \mathrm{~A}$ & $118.2(9)$ & $\mathrm{C} 6-\mathrm{C} 7-\mathrm{C} 8$ & $110.76(8)$ \\
\hline $\mathrm{C} 10-\mathrm{N} 1-\mathrm{H} 1 \mathrm{~B}$ & $121.0(9)$ & $\mathrm{C} 6-\mathrm{C} 7-\mathrm{C} 10$ & $110.30(8)$ \\
\hline $\mathrm{C} 2-\mathrm{C} 1-\mathrm{H} 1$ & $120.6(8)$ & $\mathrm{C} 8-\mathrm{C} 7-\mathrm{H} 7$ & $108.4(7)$ \\
\hline $\mathrm{C} 2-\mathrm{C} 1-\mathrm{C} 6$ & $120.62(9)$ & $\mathrm{C} 10-\mathrm{C} 7-\mathrm{H} 7$ & $108.2(7)$ \\
\hline $\mathrm{C} 6-\mathrm{C} 1-\mathrm{H} 1$ & $118.8(8)$ & $\mathrm{C} 10-\mathrm{C} 7-\mathrm{C} 8$ & $111.06(8)$ \\
\hline $\mathrm{C} 1-\mathrm{C} 2-\mathrm{H} 2$ & $119.4(8)$ & $\mathrm{C} 7-\mathrm{C} 8-\mathrm{H} 8 \mathrm{~A}$ & $106.5(8)$ \\
\hline $\mathrm{C} 3-\mathrm{C} 2-\mathrm{C} 1$ & $120.46(10)$ & $\mathrm{C} 7-\mathrm{C} 8-\mathrm{H} 8 \mathrm{~B}$ & $107.9(8)$ \\
\hline $\mathrm{C} 3-\mathrm{C} 2-\mathrm{H} 2$ & $120.1(8)$ & $\mathrm{H} 8 \mathrm{~A}-\mathrm{C} 8-\mathrm{H} 8 \mathrm{~B}$ & $108.0(11)$ \\
\hline $\mathrm{C} 2-\mathrm{C} 3-\mathrm{H} 3$ & $120.9(9)$ & $\mathrm{C} 9-\mathrm{C} 8-\mathrm{C} 7$ & $113.41(8)$ \\
\hline $\mathrm{C} 2-\mathrm{C} 3-\mathrm{C} 4$ & $119.20(10)$ & $\mathrm{C} 9-\mathrm{C} 8-\mathrm{H} 8 \mathrm{~A}$ & $110.2(8)$ \\
\hline $\mathrm{C} 4-\mathrm{C} 3-\mathrm{H} 3$ & $119.9(9)$ & $\mathrm{C} 9-\mathrm{C} 8-\mathrm{H} 8 \mathrm{~B}$ & $110.5(8)$ \\
\hline $\mathrm{C} 3-\mathrm{C} 4-\mathrm{H} 4$ & $120.6(8)$ & $\mathrm{C} 8-\mathrm{C} 9-\mathrm{H} 9 \mathrm{~A}$ & $110.4(8)$ \\
\hline $\mathrm{C} 5-\mathrm{C} 4-\mathrm{C} 3$ & $120.45(9)$ & $\mathrm{C} 8-\mathrm{C} 9-\mathrm{H} 9 \mathrm{~B}$ & $110.2(9)$ \\
\hline $\mathrm{C} 5-\mathrm{C} 4-\mathrm{H} 4$ & $119.0(8)$ & $\mathrm{C} 8-\mathrm{C} 9-\mathrm{H} 9 \mathrm{C}$ & $112.4(9)$ \\
\hline $\mathrm{C} 4-\mathrm{C} 5-\mathrm{H} 5$ & $119.9(7)$ & $\mathrm{H} 9 \mathrm{~A}-\mathrm{C} 9-\mathrm{H} 9 \mathrm{~B}$ & $105.6(12)$ \\
\hline $\mathrm{C} 4-\mathrm{C} 5-\mathrm{C} 6$ & $120.73(9)$ & $\mathrm{H} 9 \mathrm{~A}-\mathrm{C} 9-\mathrm{H} 9 \mathrm{C}$ & $108.9(12)$ \\
\hline $\mathrm{C} 6-\mathrm{C} 5-\mathrm{H} 5$ & $119.4(7)$ & $\mathrm{H} 9 \mathrm{~B}-\mathrm{C} 9-\mathrm{H} 9 \mathrm{C}$ & $109.2(12)$ \\
\hline $\mathrm{C} 1-\mathrm{C} 6-\mathrm{C} 7$ & $121.41(9)$ & $\mathrm{O} 1-\mathrm{C} 10-\mathrm{N} 1$ & $122.40(9)$ \\
\hline $\mathrm{C} 5-\mathrm{C} 6-\mathrm{C} 1$ & $118.55(9)$ & $\mathrm{O} 1-\mathrm{C} 10-\mathrm{C} 7$ & $122.52(9)$ \\
\hline $\mathrm{C} 5-\mathrm{C} 6-\mathrm{C} 7$ & $119.98(9)$ & $\mathrm{N} 1-\mathrm{C} 10-\mathrm{C} 7$ & $115.07(8)$ \\
\hline $\mathrm{C} 1-\mathrm{C} 2-\mathrm{C} 3-\mathrm{C} 4$ & $0.44(16)$ & $\mathrm{C} 5-\mathrm{C} 6-\mathrm{C} 7-\mathrm{C} 8$ & $112.46(10)$ \\
\hline $\mathrm{C} 1-\mathrm{C} 6-\mathrm{C} 7-\mathrm{C} 8$ & $-64.48(12)$ & $\mathrm{C} 5-\mathrm{C} 6-\mathrm{C} 7-\mathrm{C} 10$ & $-124.17(9)$ \\
\hline $\mathrm{C} 1-\mathrm{C} 6-\mathrm{C} 7-\mathrm{C} 10$ & $58.89(11)$ & $\mathrm{C} 6-\mathrm{C} 1-\mathrm{C} 2-\mathrm{C} 3$ & $-0.55(16)$ \\
\hline $\mathrm{C} 2-\mathrm{C} 1-\mathrm{C} 6-\mathrm{C} 5$ & $0.03(15)$ & $\mathrm{C} 6-\mathrm{C} 7-\mathrm{C} 8-\mathrm{C} 9$ & $-178.47(8)$ \\
\hline $\mathrm{C} 2-\mathrm{C} 1-\mathrm{C} 6-\mathrm{C} 7$ & $177.01(9)$ & $\mathrm{C} 6-\mathrm{C} 7-\mathrm{C} 10-\mathrm{O} 1$ & $-83.85(11)$ \\
\hline $\mathrm{C} 2-\mathrm{C} 3-\mathrm{C} 4-\mathrm{C} 5$ & $0.18(15)$ & $\mathrm{C} 6-\mathrm{C} 7-\mathrm{C} 10-\mathrm{N} 1$ & $95.67(10)$ \\
\hline $\mathrm{C} 3-\mathrm{C} 4-\mathrm{C} 5-\mathrm{C} 6$ & $-0.70(15)$ & $\mathrm{C} 8-\mathrm{C} 7-\mathrm{C} 10-\mathrm{O} 1$ & $39.34(13)$ \\
\hline
\end{tabular}


supporting information

\begin{tabular}{llll}
$\mathrm{C} 4-\mathrm{C} 5-\mathrm{C} 6-\mathrm{C} 1$ & $0.59(15)$ & $\mathrm{C} 8-\mathrm{C} 7-\mathrm{C} 10-\mathrm{N} 1$ & $-141.14(9)$ \\
$\mathrm{C} 4-\mathrm{C} 5-\mathrm{C} 6-\mathrm{C} 7$ & $-176.43(9)$ & $\mathrm{C} 10-\mathrm{C} 7-\mathrm{C} 8-\mathrm{C} 9$ & $58.60(11)$ \\
\hline
\end{tabular}

Hydrogen-bond geometry $\left(A,{ }^{\circ}\right)$

\begin{tabular}{lllll}
\hline$D-\mathrm{H} \cdots A$ & $D-\mathrm{H}$ & $\mathrm{H} \cdots A$ & $D \cdots A$ & $D-\mathrm{H} \cdots A$ \\
\hline $\mathrm{N} 1-\mathrm{H} 1 B \cdots \mathrm{O} 1^{\mathrm{i}}$ & $0.895(15)$ & $2.071(15)$ & $2.9533(13)$ & $168.4(13)$ \\
\hline
\end{tabular}

Symmetry code: (i) $x,-y+1 / 2, z+1 / 2$. 